\title{
Posterior tooth size discrepancy
}

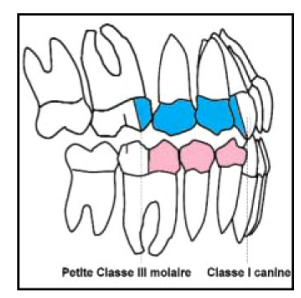

\section{Arlette OUEISS, Christine MARCHAL-SIXOU, Ahmad DALLOW, Pascal BARON, Jacques FAURE}

ABSTRACT

Despite having a correct anterior occlusal relationship between upper and lower teeth with canines in perfect Class I, some patients have imperfect posterior occlusal relationships with first molars in positions that are slightly Class II or Class III owing to an arch length discrepancy in the upper and lower buccal segments.

From this study of 62 subjects treated with full banded appliances and no bicuspid extractions we were able to determine the ideal relationship of the widths of the lower bicuspid and molar teeth with their upper antagonists, one that make it possible for the attainment of Class I relationships of both canines and first molars. This ideal value is 1.062 .

We discuss the therapeutic implications of this finding, notably its influence on extraction decisions and propose solutions for cases of frank disharmony.

\section{KEYWORDS}

Occlusal finishing

Tooth size dyscrepancy.

Address for correspondence:

J. FAURE

Faculté de

Chirurgie dentaire

(UPS, Toulouse III),

Service d'Orthopédie

dento-faciale

3, chemin

des Maraïchers,

31400 Toulouse.

faure_ja@club-internet.fr 


\section{1 - INTRODUCTION}

Ideal occlusion is defined as the simultaneous occurrence of Class I canine and Class I molar relationships, with an important additional element associated with the character of the anterior elements, that is the canine function. The tenets of occlusodontia insist on the dominant importance of canine guidance, which rigorously requires that the canines be in Class I in closure.

Class I molar relationship is seen in full inter-cuspation in centric relation when the position of the mesiopalatal cusp of the upper first molar lies in the central fossa of the lower first molar. The corresponding buccal position by classical definition requires that the mesio-buccal cusp of the upper first molar should rest in the buccal groove of the lower first molar. But many authors have adopted another definition for an ideal molar relationship: the buccal cusp of the upper second bicuspid should be inserted precisely between the lower second bicuspid and lower first molar.

In our daily clinical practice we find that the simultaneous attainment of ideal anterior and posterior relationships is far from routine.

\section{1 - 1 - Should orthodontists favor one relationship and, if so, which one?}

If one reflects on the development of the dentition, it is clear that the inter-cuspation of buccal centric occlusion establishes itself first and that the anterior occlusion confirms itself much later in an adaptation to the posterior situation that seems clearly to have priority.

But in occlusodontia, anterior guidance is the dominant element and the therapist should accord it first consideration and then help the buccal segments to come into harmony with the anterior teeth.

This means the practitioner should scrupulously regulate anterior guidance while keeping posterior teeth out of occlusion, in a "pre-eruptive" position or Tweed's post-therapeutic occlusion, which is a reversal of normal development chronology. In this conception, molar groups occlude after the anterior sector does and, it is hoped, do it in accordance with functional constraints. This is the therapeutic stance that we prefer.

\section{1 - 2 - How to analyze the frequently observed difference between an imperfect Class I molar relationship accompa- nied by a perfect Class I canine relationship?}

Unless lateral diastemas are present in one of the arches, this lack of concord in the Class I relationships of the molars and canines can be explained by a posterior tooth size discrepancy (fig. 1).

If the sum of the mesio-distal widths of the groups of upper bicuspids and molars is greater than the total over-all widths of the corresponding lower teeth, when the canine teeth are in Class I the molars will have to be in Class III, or if the 


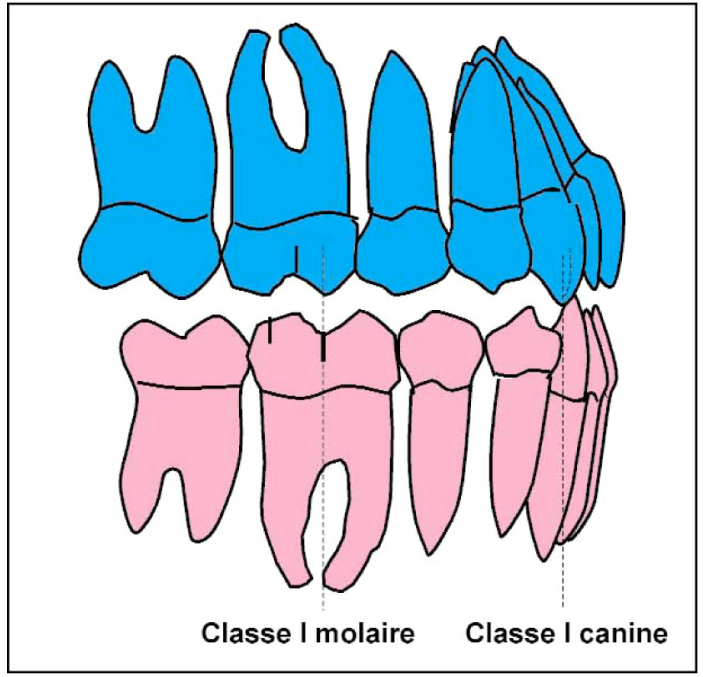

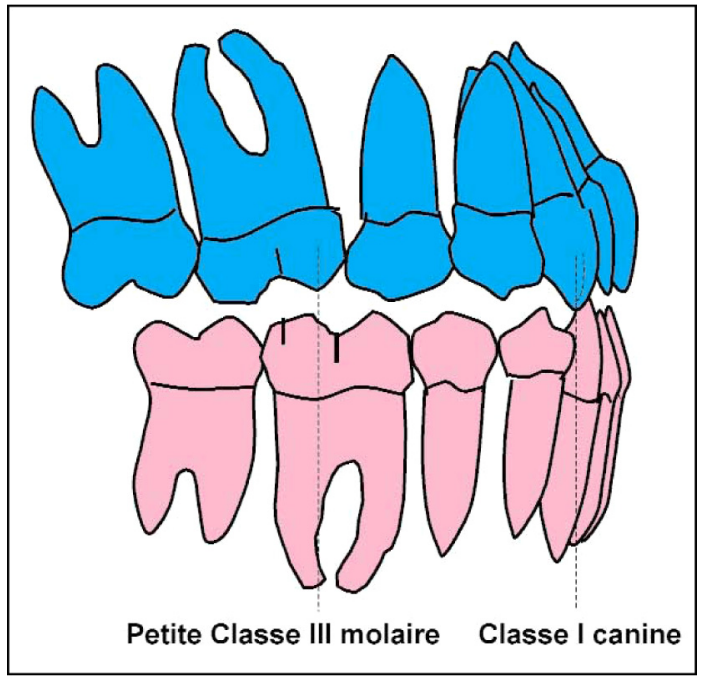

b

Figures $1 a$ and $b$

The responsibility of the posterior arch length discrepancy in the establishment of a Class III in cases of excess maxillary tooth substance;

a: occlusion with Class I molars and canines;

b: occlusion with Class I canine and "Andrew key" molar relationship.

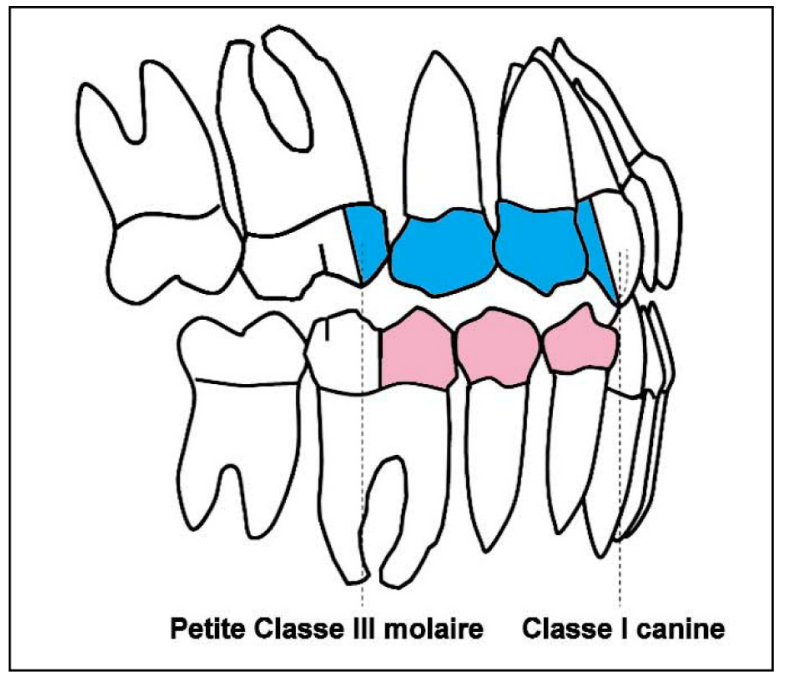

Figure 2

The upper and lower anatomic elements responsible for the lack of conformity between the Class I molar relationship and the Class I canine relationship.

molars are in Class I the canines will have to be in Class II. We believe that the first, a Class I canine and Class III molar is preferable. We often note, with some dismay, that when we have completed treatment with a well-seated Class I molar relationship, that the canines are slightly in Class II 
and that a minor overjet remains. The treating orthodontist had certainly removed the appliances without completing a finishing stage when it would have been advisable to add a stage of Class II mechanics and, doubtless, lighten the molar contacts! Some authors suggest, especially in Class II type patients, that at termination of treatment the upper molars should be left in a slight Class III relationship and angled with a forward tip as they "embrace" the lower molars.

It is surprising that this positioning is underlined proudly: if the molars occupy such a position it is because there is a slight tooth size posterior discrepancy (upper exces). It is interesting to note that the occlusion Tweed advocated, upper molars tipped back and distal cusps out of occlusion, arrives spontaneously as an Andrews key three months after removal of appliances, when there is an excess of upper tooth material (for example fig. 4)!

Therefore, the observation of an Andrews key in a finished case with perfect anterior relationships doesn't result from an exquisite treatment finishing of the occlusion, but from an anatomic accident (an excess of posterior maxillary tooth material).

\section{1 - 3 - What teeth are responsible for the arch length discrepancy that created imbalance in the anterior and poste- rior Class I relationship?}

The lack of concord between the qualities of the Class I canine and the Class I molar relationships may be related $n$ part to the distance in the maxilla between the canine cusp and the mesio-buccal cusp of the first molar and in part to the distance in the mandible between the caninebicuspid space and the buccal groove of the first molar (fig. 2). These quantities correspond respectively to these totals:

- Distal half of the canine, mesiodistal width of the premolars, mesial portion of the first molar (distance mesio-buccal cusp/mesial contact point).

- Mesio-distal widths of the premolar-mesial portion of the first molar (distance buccal groove/ mesial contact point).

In this study, the interaction between the bicuspid-molar groups is only an approximation: the complete integration of their anterior portions can be accomplished only if their morphology is not atypical.

We explain our not including the upper demi- canine in the study by our sample's presentation of uniformly perfect canine Class I, a testimony to the standardization of canine morphology.

\section{1 - 4 - What factors are involved in extraction choice when an arch length discrepancy is present?}

Some members of the orthodontic community point an accusatory finger at the extraction of first bicuspid teeth. In a view widely shared by clinicians, they affirm that the first premolar is a more important tooth in the maxillary arch than the second bicuspid, while the lower second bicuspid plays a more important functional role than does the mandibular first bicuspid. The extraction of first bicuspids in the maxilla subtracts more tooth substance 

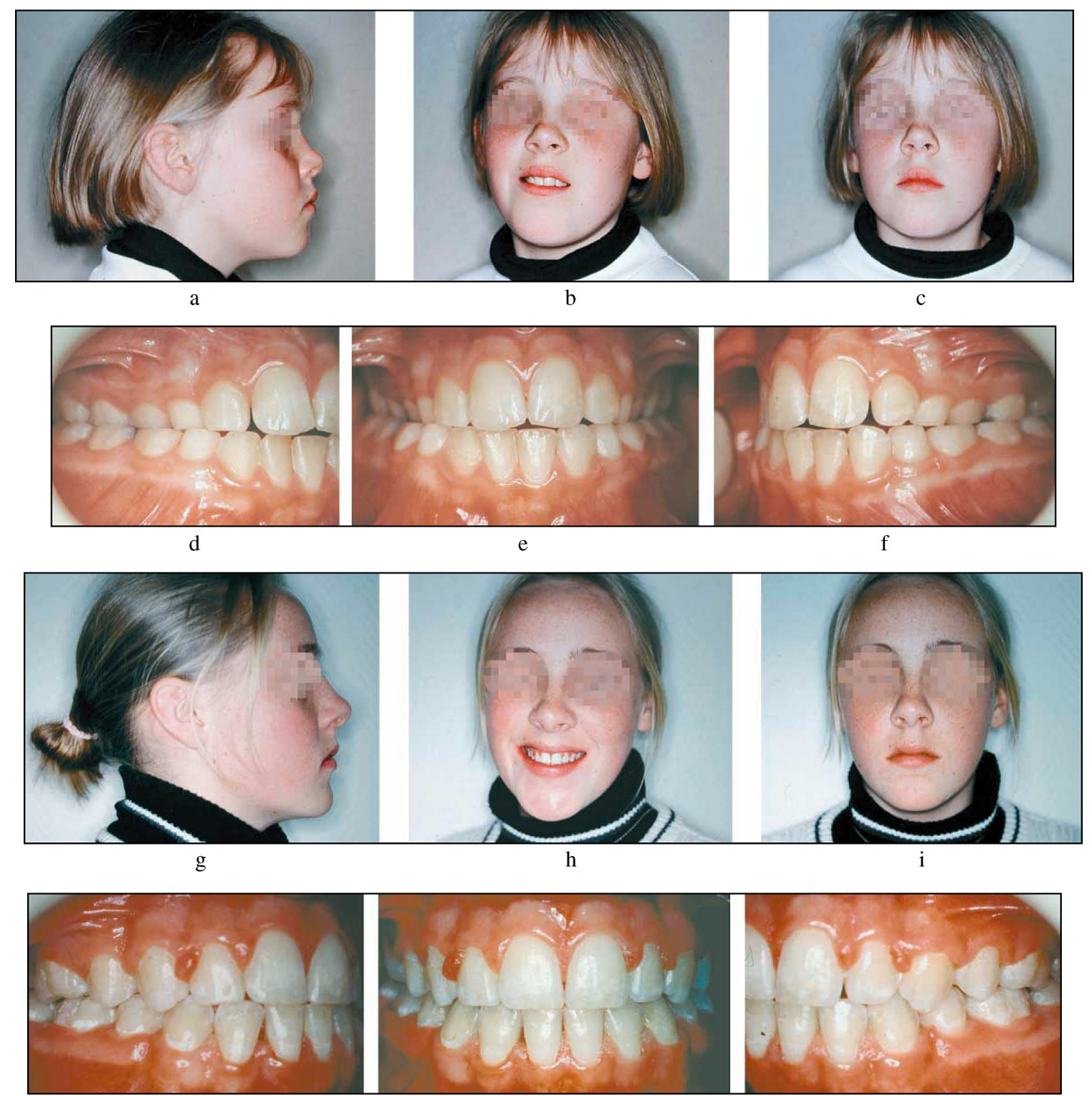

k
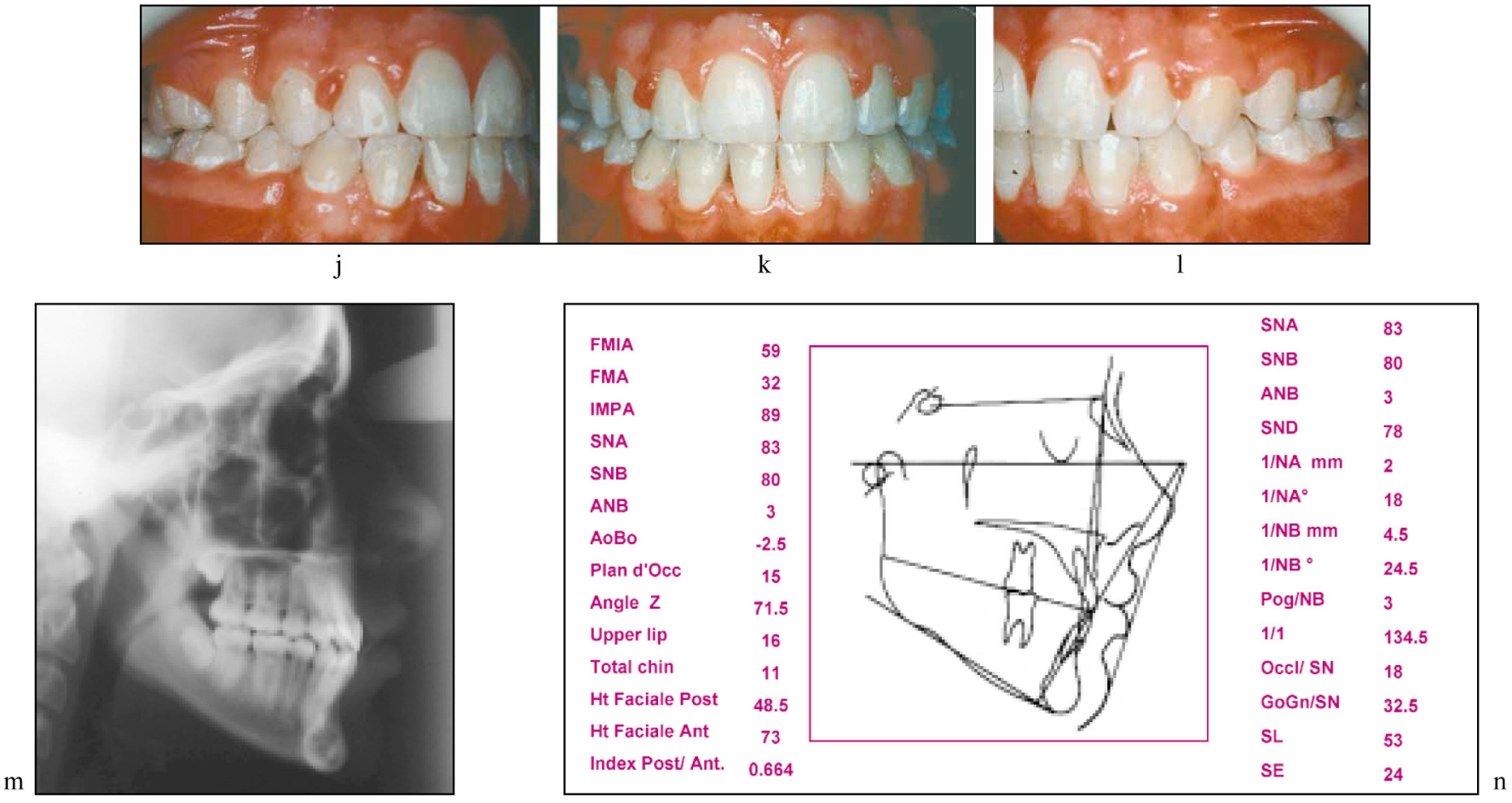

J Dentofacial Anom Orthod 2008;11:08-22. 


\begin{tabular}{|llllll|}
\hline Céphalométrie & Objectif & $\begin{array}{l}2 / 1994 \\
9 \mathrm{a} 7 \mathrm{~m}\end{array}$ & $\begin{array}{l}11 / 1995 \\
11 \mathrm{a} 4 \mathrm{~m}\end{array}$ & $\begin{array}{l}12 / 1996 \\
12 \mathrm{a} 5 \mathrm{~m}\end{array}$ & $\begin{array}{l}2 / 2000 \\
15 \mathrm{a}\end{array}$ \\
\hline \hline SNA & 82 & 83 & 82.5 & 83.5 & 83 \\
SNB & 80 & 79 & 79 & 80 & 80 \\
ANB & 2 & 4 & 3.5 & 3.5 & 3 \\
SND & 76 & 75 & 75.5 & 78 & 78 \\
1/NA mm & 4 & 4.5 & 4 & 5 & 2 \\
1/NA ${ }^{\circ}$ & 22 & 21 & 21.5 & 20 & 18 \\
1/NB mm & 4 & 5.5 & 5 & 6 & 4.5 \\
1/NB & 25 & 25.5 & 25.5 & 24.5 & 24.5 \\
Pog/NB & 1 & 0 & 0.5 & 1 & 3 \\
1/1 & 131 & 128 & 129 & 133 & 134.5 \\
Occl/SN & 14 & 20 & 19.5 & 16.5 & 18 \\
GoGn/SN & 32 & 33 & 35 & 32.5 & 32.5 \\
SL & 51 & 49 & 47.5 & 54.5 & 53 \\
SE & 22 & 21 & 21 & 25.5 & 24 \\
\hline
\end{tabular}

\begin{tabular}{|l|c|c|c|c|c|}
\hline Céphalométrie & Objectif & $\begin{array}{c}2 / 1994 \\
9 \mathrm{a} 7 \mathrm{~m}\end{array}$ & $\begin{array}{c}11 / 1995 \\
11 \mathrm{a} 4 \mathrm{~m}\end{array}$ & $\begin{array}{c}12 / 1996 \\
12 \mathrm{a} 5 \mathrm{~m}\end{array}$ & $\begin{array}{c}2 / 2000 \\
15 \mathrm{a} 7 \mathrm{~m}\end{array}$ \\
\hline FMIA & $27^{\circ}+/ / 3$ & 61 & 61.5 & 62 & 59 \\
FMA & $25^{\circ}+/-3$ & 28 & 29 & 29 & 32 \\
IMPA & $88^{\circ}+/-3$ & 91 & 89.5 & 89 & 89 \\
SNA & $82^{\circ}$ & 83 & 82.5 & 83.5 & 83 \\
SNB & $80^{\circ}$ & 79 & 79 & 80 & 80 \\
ANB & $2^{\circ}+/-2$ & 4 & 3.5 & 3.5 & 3 \\
AoBo & $2 \mathrm{~mm}+2$ & -2 & -2.5 & -3 & -2.5 \\
Plan d'Occ & $10^{\circ}$ & 12.5 & 13 & 10.5 & 15 \\
Angle Z & $75+/-5$ & 73 & 70.5 & 72.5 & 71.5 \\
Upper lip & $/$ & 11.5 & 11.5 & 11.5 & 16 \\
Total chin & $/$ & 12 & 10 & 10.5 & 11 \\
Ht Faciale Post. & $45 \mathrm{~mm}$ & 40 & 42 & 48 & 48.5 \\
Ht Faciale Ant. & $65 \mathrm{~mm}$ & 65 & 64 & 71 & 73 \\
Index Post./Ant. & 0,69 & $\mathbf{0 . 6 1 5}$ & $\mathbf{0 . 6 5 6}$ & $\mathbf{0 . 6 7 6}$ & 0.664 \\
Rapport d'évol. & $2 / 1$ & & 1.066 & 1.029 & 0.982 \\
\hline
\end{tabular}

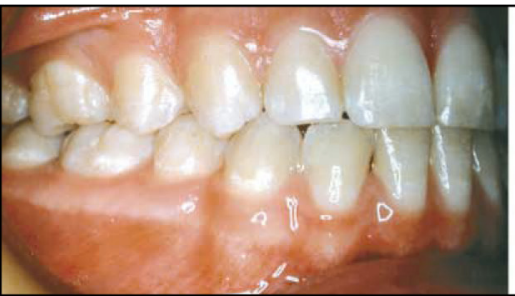

q

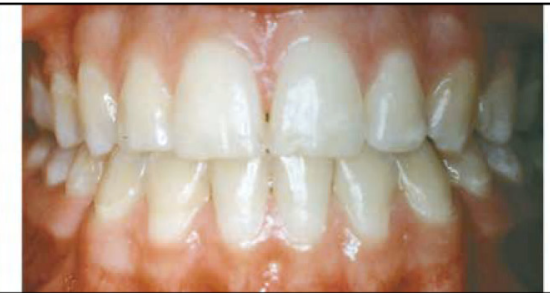

r

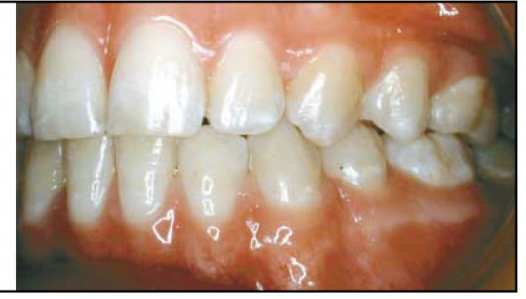

S

Figures 3 a to $s$

Management of a complex arch length discrepancy case, with a deliberate decision to finish with an "Andrews key" occlusion of the molars.

This patient, who originally had a Class III malocclusion with a complex arch length discrepancy, was treated in two stages, with extraction of the premolars (15-25-34-44) and the third molars. Thesefindings were, present:

- an anterior arch length discrepancy with excess mandibular tooth material, managed by stripping from canine to canine, finished with a much reduced anterior overbite and a rigorously maintained canine Class I relationship;

- a posterior arch length discrepancy (maxillar excess) that was left intact with no reshaping or stripping of teeth and the molars left in a slight Class III "Andrews key" relationship.

$a, b$ and $c$ : portraits at the beginning of treatment: 2/94, 9a 7m;

$d$, e and f: intra-oral views at the beginning of treatment: 2/94, 9a 7m;

$g, h$ and i: portraits at the end of the full-banded treatment: 2/2000, 15a 7m;

$j, k$ and l: intra-oral views on the day of band removal: 2/2000, 15a 7m;

$\mathrm{m}$ : headplate at the end of full-banded treatment: 2/2000, 15a $7 \mathrm{~m}$;

$n$ : cephalometric findings at the close of bull-banded treatment: 2/2000, 15a 7m;

o: cephalometric changes: Steiner analysis;

p: cephalometric changes: Tweed analysis;

$q, r$ and s: intra-oral views three years after treatment, showing good stability: 8/2003, 19a $1 \mathrm{~m}$. 


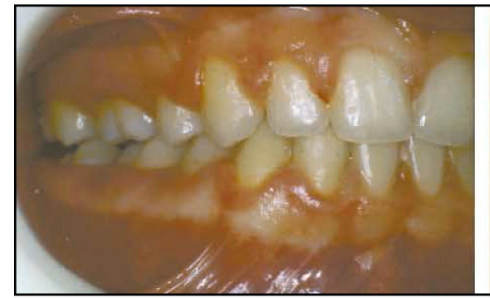

a

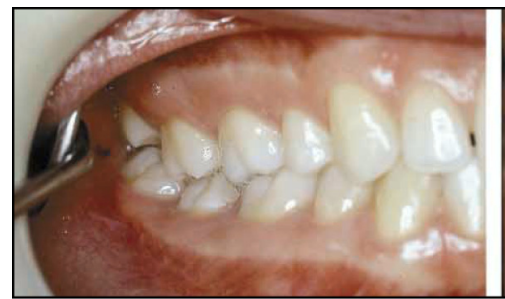

d

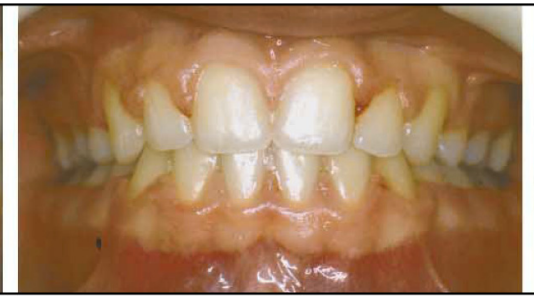

b

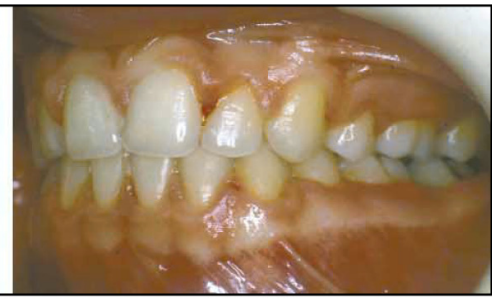

c

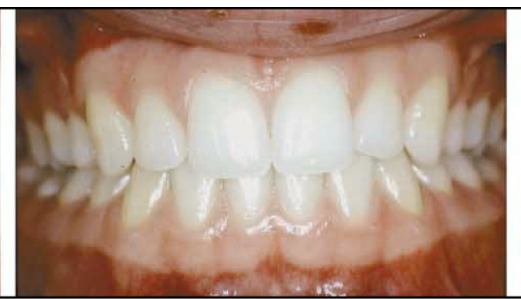

e

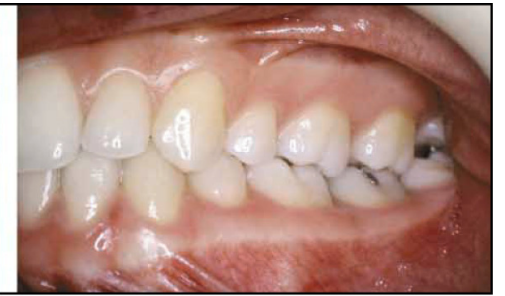

f

Figures 4 a to $f$

Undetected posterior arch length discrepancy with excess maxillary tooth material. This was a severe hyperdivergent Class II malocclusion with an arch length discrepancy caused by excess maxillary tooth structure.

A Tweed type occlusion was achieved fairly quickly as shown on the day of band removal. The rigorous Class I canine relationship remained unchanged, but the originally "pre-eruptive" molar teeth changed rapidly into a characteristic "Andrews key" relationship:

$a, b$ and $c$ : intra-oral views on the day of band remo- val;

$d$, e and f: intra-oral views five years after treatment. The extractions (14-24-34-44) did not play a role one way or another in the arch length discrepancy caused by an excess of posterior maxillary tooth material. This case was present in a thorough fashion in the Revue d'Orthopédie Dento-Faciale, 2005;39:449-76.

than would removal of second bicuspids, but less in the mandible. The extraction of mandibular first bicuspids, therefore, would effect an excess of lower tooth structure and contribute to a Class II tendency. The sacrifice of lower second bicuspids would have the opposite effect.

So a selective variation of extraction candidates would be less disturbing to molar relationships than extraction of the same tooth in each quadrant. But the literature does not agree in a confirmation of the existence of significant differences $4,5,78,9,11$.

\section{1 - 5 - Should we make} harmonious extraction choices routinely?

If it is clear that an arch length discrepancy is responsible for the nonconformance of the quality of the Class I relationship in the canine and molar regions, its extent must be measured and corrected when it is large:

- by augmentation in the form of a prosthesis or a restoration;

- by reduction through interproximal stripping; 
- by appropriate selective extractions.

Considerable interest has been evidenced in the literature in inciso-canine relationships and ways of managing anterior lack of har- mony $1,2,3,4,5,6,10$. But studies including the posterior region are,

\section{2 - MATERIALS AND METHODS}

\section{2 - 1 - Material and the measurement method}

Our study sample consisted of 62 nonextraction cases treated with a multiband appliance.

We obtained the records from an orthodontic office whose three practitioners have all earned certificates of excellence or from the ToulouseRangueil Department of Orthodontics for cases students had presented for the CECSMO national examination.

The criterion for inclusion in the study was a standard occlusion with perfect Class I canine relationship and normal incisor positioning that remained stable in the post-treatment period.

The criteria for exclusion were:

- extractions of bicuspids or anterior teeth. Cases with third molar extractions were accepted;

- a non-standard terminal occlusion, usually documented to have been the result of lack of cooperation as documented in the administrative record.

We measured the mesio-distal diameters of all the teeth with a calipers that was accurate to $0.05 \mathrm{~mm}$. to date, quite rare ${ }^{1,2}$. Very few authors have considered the occlusal consequences of tooth to tooth inharmony ${ }^{5}$. Accordingly, we concluded that an investigation into the relationship between arch length discrepancy and occlusal malrelationships would be worthwhile.

\section{2 - 2 - Mathematical method of evaluating the optimal Rpi relationship}

We evaluated the optimal Rpi value of the relationship of the six lower posterior teeth with the six upper posterior teeth 6PL6PU with three different methods:

- an "anatomic" based on the ideal relationships defined by Bolton for the anterior region, Rai, and for the entire dentition Rti;

- an "average anatomic" that used as an average value of the relationship $\mathrm{Rp}=6 \mathrm{PI} / 6 \mathrm{PS}$ as a reference base;

- an occlusal that examined the relationship between the Class I qualities of the canines and the molars.

\section{- An "anatomic" based on the Bolton Index}

The Bolton Index is defined as the ideal relationship between anterior groups of six teeth $R a=6 L A / 6 U A$ ) and the complete dentition, with the second molars excluded, $\mathrm{Rt}=12 \mathrm{~L} / 12 \mathrm{U}$.

The precise definition of the anterior and posterior occlusal relationships depends on a pre-determination of four dimensional parameters: 6LA, 
6UA, 6LP, 6UP (or possibly 6LA, 6UA, $12 \mathrm{~L}, 12 \mathrm{U}$ because 6LP=12L-6LA and $6 U P=12 U-6 U A)$. If one of these parameters is known, the values of the three others can be calculated by using the relationships $\mathrm{Ra}$ and $\mathrm{Rp}$ (or possibly $\mathrm{Rt}$ ), but in addition the relationship of anterior dentition/total lower dentition or of anterior dentition /posterior dentition must be added. We use the relationship anterior denture/total lower denture, RatL $=6 \mathrm{LA} / 12 \mathrm{~L}$, or upper, RatU $=6 \mathrm{UA} /$ $12 \mathrm{U}=$ Rt.RatL/Ra.

We at first studied the relationship between the parameters and the posterior occlusal relationships and defined the ideal relationships RatUi or RatLi.

For the theoretical reason we have already stated the relationship of the six lower posterior teeth to the six upper posterior teeth, 6LP/6UP, cannot be expressed solely with the help of the Bolton indices Ra et Rt, the relationship of the anterior dentition to the total must also be included.

$$
\begin{aligned}
& \frac{6 \mathrm{PI}}{6 \mathrm{PS}}=\frac{12 \mathrm{I}-6 \mathrm{AL}}{12 \mathrm{~S}-6 \mathrm{AS}}=\frac{\text { Rt. } 12 \mathrm{~S}-\mathrm{Ra} .6 \mathrm{AS}}{12 \mathrm{~S}-6 \mathrm{AS}}=\frac{\text { Rt }- \text { Ra.RatS }}{1-\text { RaS }} \\
& \frac{\mathrm{GPI}}{\text { GPS }}=\frac{\mathrm{Ri}-\mathrm{Ru} \cdot \mathrm{RaI}}{1-\mathrm{RLRat} / \mathrm{Ra}}
\end{aligned}
$$

A dentition in equilibrium is defined as one in which there is an ideal relationship between its anterior elements and its totality, Rai and Rti. With a determination of the ideal relationships RatUi or RatLi, a calculation of the corresponding ideal value Rpi can be made.

\section{- The average anatomic method}

With a calculation of the sums of the diameters of the posterior teeth (4-5-6) of the two arches we were able to determine the average dimensional relationship, which should cor- respond to what would be expected in a dentition in equilibrium.

\section{- Occlusal method seeking accord between the Class I of the canines and the Class I of the molars}

The dentitions of our study sample were in perfect Class I canine relationship but their molar relationships had changed from a moderate Class II to a moderate Class III. The relationship of the over-all dimensions of the group of upper posterior teeth to the over-all dimensions of the group of lower posterior teeth, $\mathrm{Rp}=6 \mathrm{LP} / 6 \mathrm{UP}$, pre-disposed a priori the final molar relationships. A study of the relationships between the extent of the molar Class II and the Rp relationship would make it possible to determine the optimal occlusal relationship, that is one capable assuring both a Class I canine and a Class I molar intercuspation.

\section{2 - 3 - Statistical methods}

We calculated the averages and the standard deviations of the mesiodistal widths of each tooth. We described the distribution, average and standard deviation, of the dental groups being considered, 6UA, 6LA, 6 UP, 6LP, 12U, 12L, as well as the distribution of the significant relationships, Ra, Rp, Rt and the ante- rior/ total: RatU and RatL, were evaluated.

We also assessed the significant character of the differences between first and second bicuspids in the two arches by means of a Student t test.

The correlation coefficients of the type of molar relationship with RatU and RatL, measured by a t test, were 
significant after the values 0.245/ $0.315 / 0.391$ with respective confidence levels of $5 \% / 1 \% / 1 \%$. With regressions we were able to more effectively pinpoint the optimum value for $\mathrm{Rp}$.

The regression rate of the molar class with respect to the $\mathrm{Rp}$ relationship precisely defines the ideal value for this relationship.

\section{3 - RESULTS}

\section{3 - 1 - "Anatomic" method based on the Bolton indices}

The correlation coefficient between the over-all occlusal Class III (Right + Left) and the relationship RatL is highly significant. The rate of regression shows that the Class I is acquired by
For this study we re-grouped the right and left observations and analyzed the variation of unilateral $\mathrm{Rp}$ with the extent of the corresponding Class II relationship. The sample consisted of 124 individuals. The significance thresholds for $r$ were: $0.174 / 0.227 / 0.288$ at the same confidence levels.
RatLi $=0.4133$ (fig. 5), very close to the other reference, the average value of RatL.

The correlation with RatU is not significant.

The correlations of $\mathrm{Rp}$ with RatL or RatU show the role that the relationship between the anterior dentition and over-all dentition plays in

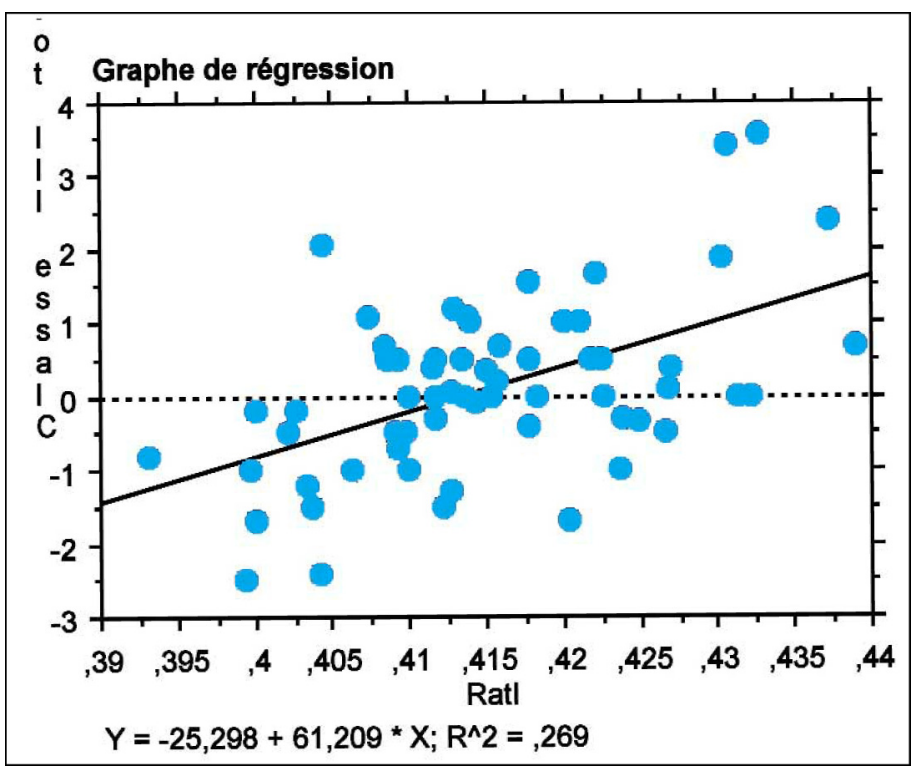

Figure 5

Regression graph of the molar relationship (the extent of the Class III) as a function of the anterior den- tition/total lower dentition relationship (Ratl).

The correlation coefficient is highly significant (0.519); the value corresponding to Class I is 0.4133 . 


\begin{tabular}{|l|c|c|}
\hline & Mean & Stand. Dev. \\
\hline Ra & 0,7638 & 0,0216 \\
$\mathrm{Rt}$ & 0,9154 & 0,0184 \\
$\mathrm{Rp}$ & 1,0658 & 0,0322 \\
RatS / RatU & 0,4975 & 0,0085 \\
Ratl / RatL & 0,4151 & 0,01 \\
\hline
\end{tabular}

Table I

Averages and standard deviations of the study sample:

Ra: 6LA/6UA;

Rt: 12L/12U;

Rp: 6LP/6UP;

RatU: 6UA/12U;

RatL: 6LA/12L.

\begin{tabular}{|c|c|c|c|c|c|}
\hline & Mean & Stand. Dev. & & Mean & Stand. Dev. \\
\hline 16 & 9,826 & 0,465 & 46 & 10,875 & 0,687 \\
15 & 6,57 & 0,477 & 45 & 7 & 0,438 \\
14 & 6,854 & 0,396 & 44 & 6,956 & 0,425 \\
13 & 7,754 & 0,544 & 43 & 6,68 & 0,406 \\
12 & 6,773 & 0,542 & 42 & 5,733 & 0,361 \\
11 & 8,594 & 0,443 & 41 & 5,185 & 0,296 \\
21 & 8,6 & 0,509 & 31 & 5,243 & 0,29 \\
22 & 6,81 & 0,497 & 32 & 5,764 & 0,369 \\
23 & 7,727 & 0,427 & 33 & 6,706 & 0,4 \\
24 & 6,892 & 0,407 & 34 & 7,031 & 0,38 \\
25 & 6,68 & 0,476 & 35 & 6,985 & 0,415 \\
26 & 9,888 & 0,467 & 36 & 10,914 & 0,621 \\
\hline 6AS/6UA & 46,258 & 2,358 & $6 A I / 6 L A$ & 35,31 & 1,681 \\
$12 S / 12 U$ & 92,968 & 4,332 & $12 \mathrm{I} / 12 L$ & 85,073 & 3,754 \\
3PSD/3RUP & 23,25 & 1,116 & 3PID/3RLP & 24,831 & 1,251 \\
3PSG/3LUP & 23,46 & 1,175 & 3PIG/3LLP & 24,931 & 1,221 \\
6PS/6UP & 46,71 & 2,255 & $6 \mathrm{PI} / 6 L P$ & 49,762 & 2,442 \\
\hline
\end{tabular}

Table /l

Averages and standard deviations of the study sample for the different teeth and the different groups of teeth:

- anterior groups (6UA and 6LA);

- arches (12U et 12L);

- right and left posterior groups (respectively 3RUP and $3 R L P / 3 L U P$ and $3 L L P$ );

- complete posterior groups (6UP and 6LP). 
the occlusal relationships of the posterior teeth, respectively $r=$ -0.497 and $r=0.357)$.

The average values for RatL and RatU are respectively 0.4151 and 0.4975 (table I).

The recording of these values in the 1 and 2 formulas by means of the ideal Bolton values for Rai and Rti make it possible to calculate the Rpi.

From the formulas 1 and 2 we get, res- pectively, for the Rp relationship.

Rpi $=1.0526$

and

Rpi= 1,0489

\section{3 - 2 - "Anatomic average" method}

The relationships of the sums of the diameters 4-5-6 on the lower with the upper give (table II):

Rpi $=1.0658+/-0.0322$

\section{3 - 3 - Occlusal method seeking an accord between Class I canine and Class I molar}

The equation or the regression rate immediately records a perfect Class I (fig. 6):

Rpi= 1.0699

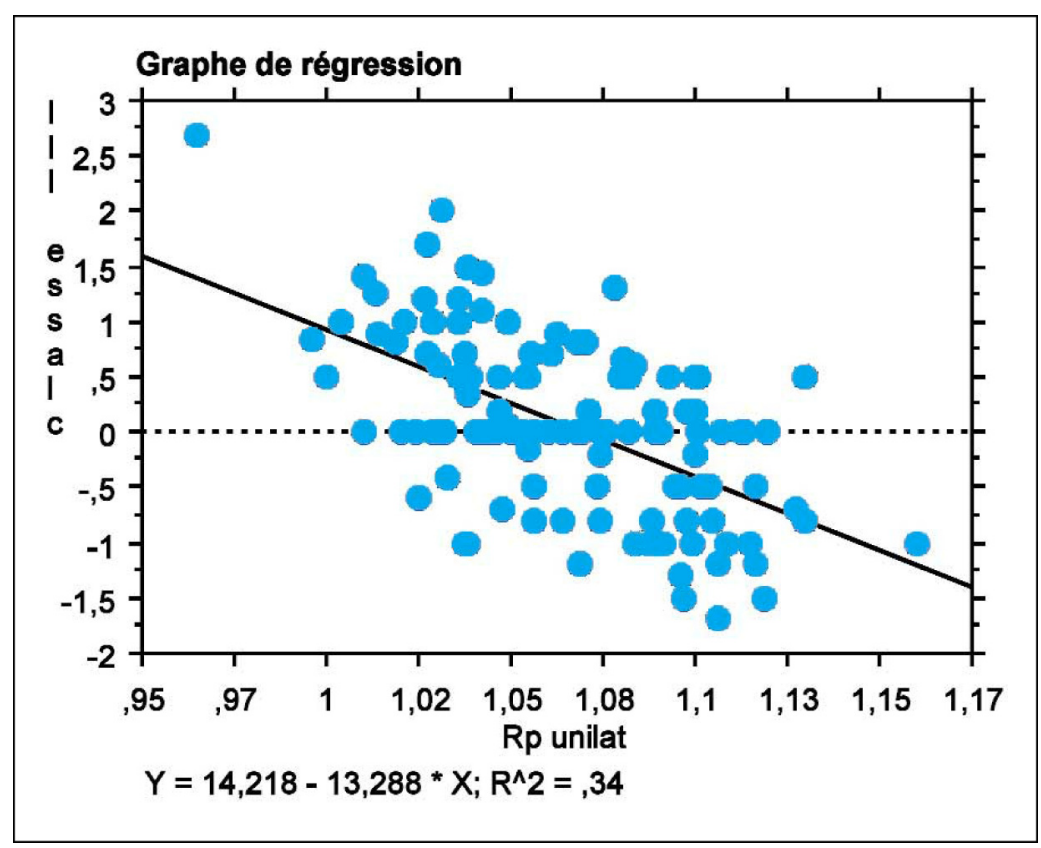

Figure 6

Regression graph of the molar relationship (the extent of the Class III) as a function of the lower posterior dentition/upper posterior dentition (Rp).

Le correlation coefficient is highly significant (-0.583); the value corresponding to Class I is 1.0699 . 
4 - DISCUSSION

\section{4 - 1 - Criticism of the method}

The observation of an average anterior rela- tionship Ra, close to the Bolton value, confirms the orthomorphic nature of the sample table II). The weak observed standard deviation type is related to patients having a perfect Class I canine, making it impossible for the Bolton Index to vary much from the ideal value.

We have already criticized the method we adopted utilizing the sum of the mesio-distal diameters of the premolars and the first molar for calculating the Rp. The highly significant correlations between Class II occlusal and Rp show that the approximation adopted was justified.

\section{4-2- The role of the choice of extractions of bicuspids in the creation or correc- tion of a posterior arch length discrepancy}

The mesio-distal diameters we recorded are very close to those cited in the literature $78,9,11$; the difference between the first and second premolars is significant in the maxilla $(0.248 \mathrm{~mm}, \mathrm{t}=8.847)$ but it is not in the mandible.

These results support the conception that choosing the upper right and left first bicuspids for extraction contributes to an excess of maxillary tooth substance and a Class II molar relationship while selection of the upper right and left second bicuspids leads to a deficit in maxillary tooth material and a Class III molar relationship.

\section{4 - 3 - What value should the Rpi have?}

The average of the three determinations, very close to each other, can be kept for reference:

$$
\text { Rpi }=\mathbf{1 . 0 6 2}
$$

\section{4 - 4 - What clinical considera- tions should this study have for practitioners?}

At the beginning of treatment, orthodontists should review morphology of premolars and molars with considerable vigilance, in order to make a de visu assessment of the possible presence of any substantial posterior arch length discrepancy. This does not mean that they should routinely and systematically measure the mesio-distal widths of all the teeth to calculate the Ra, Rp and Rt.

Unfortunately, in actual practice orthodontists usually do not discern a posterior arch length discrepancy problem until they have finished the bulk of treatment and are approa- ching the finishing stage, the warning sign being a lack of concordance between the Class I relationships in the canine and in the molar areas. Most often practitioners rejoice in having obtained a frank Class I molar relationship and then desperately try to improve the canine situation, which is slightly Class II with a modest remaining anterior overjet or the opposite, an end-to-end anterior occlusion. More rarely the anterior relationships are perfect but the molars seem stuck in a slight Class II or modest Class III. 
At that time the orthodontist ought to quantify the arch length discrepancy by calculating the Rp index, which helps to assess the excess of tooth substance in one arch or deficit in the other. Thus in the present an increased $\mathrm{Rp}$, the orthodontist can either:

- consider the inferior arch to be correct and then calculate the ideal maxillary value: $6 \mathrm{UPi}=$ 6LP/Rpi and thus the upper deficit would be: 6UPi-6UP;

- consider the superior arch to be correct and then calculate the ideal mandibular value: $6 \mathrm{LPi}=$ 6UPxRpi and thus the mandibular excess: 6LP-6LPi.

The primary therapeutic objective is the establishment and the comfortable implementation of a canine Class I and of perfect anterior guidance. As for the posterior relationships, which would then be out of kilter, the therapeutic stance to adopt would depend upon the severity of the arch length discrepancy and the patient's willingness to undergo additional treatment.

- In the pursuit of an ideal treatment result, the orthodontist would correct the lack of morphological harmony if possible

\section{5 - CONCLUSION}

This study emphasizes the importance of posterior arch length discrepancy and proposes a measurement tool, the posterior lower to upper relationship $R p=6 L P / 6 U P($ reference value: 1.062).

Lack of posterior harmony is responsible for instances of non-concordance of the Class I relationships of the canines and the molars. by removing inter-proximal enamel by stripping or, effectively, increasing enamel mass with composite fillings, remaking prostheses, or appropriately adapting mesio-occlusal and mesio-distal restorations.

- By accepting an imperfect Class I molar relationship but rigorously adjusting the occlusion in certain cases, for example a modest Class III molar relationship in a patient with a Class III type face to secure a stable occlusion (fig. 3), especially when anterior occlusion is perfect.

- When the observed imperfections are indications of over-correction, for example, a modest Class III finish of a mal-occlusion that was originally Class II (fig. 4), this situation is "no risk". The orthodontist has only to adjust the anterior occlusion scrupulously and the molars will spontaneously seat themselves in accordance with this optimal compromise of and Andrews key to occlusion.
Confronted with this clinical situation, our therapeutic choice is unequivocally to aim at achieving a perfect anterior guidance:

- either by accepting a slight imperfection in the posterior relationships as would be expected by the $\mathrm{Rp}$ reading;

- or by changing the shape and size of selected posterior teeth. 


\section{REFERENCES}

1. Bolton W.A. Disharmony in tooth size and its relation to the analysis and treatment of malocclusion. Am J Orthod 1958;28:113-30.

2. Bolton W.A. The clinical application of tooth size analysis. Am. J Orthod 1952;48:504-29.

3. Faure J, Baron P. Troubles morphologiques du bloc incisif supérieur. J Edge 1997;36:78-88.

4. Freeman J.E, Maskeroni A.J, Lorton L. Frequency of Bolton tooth size discrepancy among orthodontic patients. Am. J Orthod 1996;110:24-7.

5. Grosby D.R, Alexander C.G. The occurrence of tooth size discrepancy among different malocclusion groups. Am. J Orthod 1989;95:186-9.

6. Lorette M.A. Dysharmonies dento-basales et dento-dentaires. Orthod. Fr 1975;46:177-84.

7. Marseiller E. Les dents humaines: morphologie. Gauthier-Villars éditeur. 1969.

8. Nabbout F, Faure J, Baron P, Braga J, Treil J. Anatomie dentaire et orthodontie. L'apport du scanner 3D/Dental anatomy and orthodontics. The benefit of the 3D scanner. Rev Orthop Dento Faciale 2003;37:59-73.

9. Nabbout F, Faure J, Baron P, Braga J, Treil J. L'ancrage dentaire en orthodontie: les données du scanner/Tooth anchorage in orthodontics: scanner generated data. International Orthod 2004;2:241-56.

10. Pujol A. Contribution à l'étude des dysharmonies dento-dentaires. Thèse $3^{\circ}$ cycle Sci. Odontol. Bordeaux. 1977; n43047708.

11. Romorovski J, Bresson G. Morphologie dentaire de I'adulte. EMC Editions techniques. Stomatologie 1994;22003(A-10):34 pages.

\section{Abbreviations employed in this study:}

6LA: sum of the mesio-distal widths of the lower incisor-canine group (43; $42 ; 41 ; 31 ; 32 ; 33)$

$6 U A$ : sum of the mesio-distal widths of the upper incisor-canine group (13; $12 ; 11 ; 21 ; 22 ; 23)$

12L: sum of the mesio-distal widths of the teeth between the two lower first molars $(46 ; 45 ; 44 ; 43 ; 42 ; 41 ; 31 ; 32 ; 33 ; 34 ; 35 ; 36)$

$12 \mathrm{U}$ : sum of the mesio-distal widths of the teeth between the two upper first molars $(16 ; 15 ; 14 ; 13 ; 12 ; 11 ; 21 ; 22 ; 23 ; 24 ; 25 ; 26)$

6LP: sum of the mesio-distal widths of the lower premolar-molar group (46; $45 ; 44 ; 34 ; 35 ; 36)$

6UP: sum of the mesio-distal widths of the lower premolar-molar group (16; $15 ; 14 ; 24 ; 25 ; 26)$

Ra: 6LA/6UA

Rt: 12L/12U

Rp :6LP/6UP

RatU: 6UA/12U

RatL: 6LA/12L 